\title{
ON ESTIMATES FOR THE WEIGHTS IN GAUSSIAN QUADRATURE IN THE ULTRASPHERICAL CASE
}

\author{
KLAUS-JÜRGEN FÖRSTER AND KNUT PETRAS
}

ABstRaCT. In this paper the Christoffel numbers $a_{\nu, n}^{(\lambda) G}$ for ultraspherical weight functions $w_{\lambda}, w_{\lambda}(x)=\left(1-x^{2}\right)^{\lambda-1 / 2}$, are investigated. Using only elementary functions, we state new inequalities, monotonicity properties and asymptotic approximations, which improve several known results. In particular, denoting by $\theta_{\nu, n}^{(\lambda)}$ the trigonometric representation of the Gaussian nodes, we obtain for $\lambda \in[0,1]$ the inequalities

$$
\begin{aligned}
& \frac{\pi}{n+\lambda} \sin ^{2 \lambda} \theta_{\nu, n}^{(\lambda)}\left\{1-\frac{\lambda(1-\lambda)}{2(n+\lambda)^{2} \sin ^{2} \theta_{\nu, n}^{(\lambda)}}\right\} \\
& \leq a_{\nu, n}^{(\lambda) G} \leq \frac{\pi}{n+\lambda} \sin ^{2 \lambda} \theta_{\nu, n}^{(\lambda)}
\end{aligned}
$$

and similar results for $\lambda \notin(0,1)$. Furthermore, assuming that $\theta_{\nu, n}^{(\lambda)}$ remains in a fixed closed interval, lying in the interior of $(0, \pi)$ as $n \rightarrow \infty$, we show that, for every fixed $\lambda>-1 / 2$,

$$
\begin{aligned}
a_{\nu, n}^{(\lambda) G}=\frac{\pi}{n+\lambda} \sin ^{2 \lambda} \theta_{\nu, n}^{(\lambda)}\{ & 1-\frac{\lambda(1-\lambda)}{2(n+\lambda)^{2} \sin ^{2} \theta_{\nu, n}^{(\lambda)}} \\
& \left.-\frac{\lambda(1-\lambda)\left[3(\lambda+1)(\lambda-2)+4 \sin ^{2} \theta_{\nu, n}^{(\lambda)}\right]}{8(n+\lambda)^{4} \sin ^{4} \theta_{\nu, n}^{(\lambda)}}\right\}+O\left(n^{-7}\right) .
\end{aligned}
$$

\section{INTRODUCTION}

For fixed real $\lambda>-1 / 2$ let $w_{i}$ be the ultraspherical weight function

$$
w_{\lambda}(x):=\left(1-x^{2}\right)^{\lambda-1 / 2}, \quad x \in(-1,1),
$$

and let $P_{n}^{(\lambda)}$ be the corresponding orthogonal polynomial of degree $n$ normalized by $P_{n}^{(\lambda)}(1)=\left(\begin{array}{c}n+2 \lambda-1 \\ n\end{array}\right)$. Furthermore, let $x_{1, n}^{(\lambda)}<x_{2, n}^{(\lambda)}<\cdots<x_{n, n}^{(\lambda)}$ be the zeros of $P_{n}^{(\lambda)}$ in increasing order and let $0<\theta_{1, n}^{(\lambda)}<\theta_{2, n}^{(\lambda)}<\cdots<\theta_{n, n}^{(\lambda)}<\pi$ be their trigonometric representation $x_{\nu, n}^{(\lambda)}=-\cos \theta_{\nu, n}^{(\lambda)}$.

Received January 13, 1989; revised August 1, 1989.

1980 Mathematics Subject Classification (1985 Revision). Primary 65D30, 41 A55.

Key words and phrases. Christoffel numbers, Gaussian quadrature. 
In this paper we consider quadrature formulae $Q_{n}$ of (algebraic) degree $\operatorname{deg}\left(Q_{n}\right)=m \geq 0$ which are linear functionals of the type

$$
\begin{gathered}
Q_{n}[f]:=\sum_{\nu=1}^{n} a_{\nu, n} f\left(x_{\nu, n}\right), \quad-1 \leq x_{1, n}<x_{2, n}<\cdots<x_{n, n} \leq 1, \\
\int_{-1}^{1} f(x) w_{\lambda}(x) d x=Q_{n}[f]+R_{n}[f], \\
R_{n}\left[p_{\mu}\right] \begin{cases}=0 & \text { for } \mu=0, \ldots, m, \\
\neq 0 & \text { for } \mu=m+1,\end{cases}
\end{gathered}
$$

where the $n$ nodes $x_{\nu, n}$ and the weights $a_{\nu, n}$ are real numbers, $p_{\mu}$ denotes the monomial $p_{\mu}(x)=x^{\mu}$, and $R_{n}$ is the so-called 'error functional' of $Q_{n}$. Of all quadrature rules that have received attention, Gaussian quadrature is the most investigated (cf. Gautschi [19] and the bibliography cited therein). The Gaussian quadrature formula $Q_{n}^{(\hat{\lambda}) G}$,

$$
Q_{n}^{(\hat{\lambda}) G}[f]:=\sum_{\nu=1}^{n} a_{\nu, n}^{(\lambda) G} f\left(x_{\nu, n}^{(\lambda) G}\right)
$$

is determined uniquely by its having maximal degree $\operatorname{deg}\left(Q_{n}^{(\lambda) G}\right)=2 n-1$. The respective nodes $x_{\nu, n}^{(\lambda) G}$ are the zeros of $P_{n}^{(\lambda)}$, and the respective weights $a_{\nu, n}^{(\lambda) G}$, the so-called 'Christoffel numbers', are given by

$$
a_{\nu, n}^{(\lambda) G}=\frac{2^{2-2 \lambda} \pi \Gamma(n+2 \lambda)}{[\Gamma(\lambda)]^{2} \Gamma(n+1)}\left[1-\left(x_{\nu, n}^{(\lambda) G}\right)^{2}\right]^{-1}\left[P_{n}^{(\lambda)^{\prime}}\left(x_{\nu, n}^{(\lambda) G}\right)\right]^{-2}, \quad \lambda \neq 0
$$

(cf. Szegö [36, §15.3]). By the symmetry of the weight function $w$, the Gaussian formula $Q_{n}^{(\lambda) G}$ is symmetric, i.e., $x_{\nu, n}^{(\lambda) G}=-x_{n+1-\nu, n}^{(\lambda) G}$ and $a_{\nu, n}^{(\hat{\lambda}) G}=$ $a_{n+1-\nu, n}^{(\lambda) G}(\nu=1, \ldots, n)$. Therefore, it suffices to consider $\nu=1,2, \ldots$, $[(n+1) / 2]$. In particular, we have that

$$
x_{(n+1) / 2, n}^{(i) G}=0, \quad a_{(n+1) / 2, n}^{(i) G}=\frac{\pi}{2} \frac{\Gamma\left(\frac{n}{2}+\frac{1}{2}\right) \Gamma\left(\frac{n}{2}+\lambda\right)}{\Gamma\left(\frac{n}{2}+1\right) \Gamma\left(\frac{n}{2}+\lambda+\frac{1}{2}\right)} \text { for odd } n .
$$

If $\lambda \notin\{0,1\}$, for the other nodes $x_{\nu, n}^{(\lambda) G}$ and weights $a_{\nu, n}^{(\lambda) G}$, elementary representations as in (1.6) are not known, but the knowledge of their asymptotic behavior and of explicit upper and lower bounds may be important for various theoretical and practical investigations in quadrature; see, e.g., the monographs on quadrature of Braß [5] and Davis and Rabinowitz [10] as well as the remarks in $\S 5$.

For the nodes $x_{\nu, n}^{(i)(i}$ several authors have obtained inequalities or asymptotic approximations; see, e.g., Szegö [36, pp. 116 ff], Tricomi [37], Ahmed, Muldoon, and Spigler [2] and, in particular, Gatteschi [17]. For Gaussian weights 
$a_{\nu, n}^{(\lambda) G}$ it is well known (cf. Szegö [36, §15.3] and Davis and Rabinowitz [10]) that

$$
a_{\nu, n}^{(\lambda) G}=\frac{\pi}{n+\lambda} \sin ^{2 \lambda} \theta_{\nu, n}^{(\lambda)}\left(1+\delta_{\nu, n}^{(\lambda) G}\right), \quad \lim _{n \rightarrow \infty} \delta_{\nu, n}^{(\lambda) G}=0
$$

provided that the nodes $x_{\nu, n}^{(\lambda) G}$ remain in a fixed closed interval lying in the interior of $[-1,1]$ and that $\lambda$ is fixed. Whitney [38] improved (1.7) by showing the asymptotic estimate (cf. also Ossicini [28])

$$
\left|\delta_{\nu, n}^{(\lambda) G}\right| \leq \frac{|\lambda(\lambda-1)|}{(n+\lambda)^{2} \sin ^{2} \theta_{\nu, n}^{(\lambda)}}\left(1+\widetilde{\delta}_{\nu, n}^{(\lambda) G}\right), \quad \lim _{n \rightarrow \infty} \widetilde{\delta}_{\nu, n}^{(\lambda) G}=0, \quad \lambda>0,
$$

valid under the same conditions on $\nu$ and $\lambda$ as above. Recently, Gatteschi [18] obtained the following important asymptotic approximation, which is uniform with respect to the parameter $\nu=1,2, \ldots,[q n]$ for fixed $q \in(0,1)$ as $n \rightarrow \infty$,

$$
\begin{aligned}
a_{\nu, n}^{(\lambda) G}= & \frac{2}{n+\lambda} \frac{\sin ^{2 \lambda} \theta_{\nu, n}^{(\lambda)}}{j_{\lambda-1 / 2, \nu} J_{\lambda+1 / 2}^{2}\left(j_{\lambda-1 / 2, \nu}\right)} \\
& \cdot\left\{1-\frac{\lambda(1-\lambda)}{2(n+\lambda)^{2}}\left(\sin ^{-2} \theta_{\nu, n}^{(\lambda)}-\left(\theta_{\nu, n}^{(\lambda)}\right)^{-2}\right)+\theta_{\nu, n}^{(\lambda)} O\left(n^{-3}\right)\right\},
\end{aligned}
$$

where $j_{\alpha, k}$ is the $k$ th positive zero of the Bessel function $J_{\alpha}$. Shohat and Winston [31] proved

$$
a_{\nu, n}^{(\lambda) G}>\frac{1}{n+\lambda+\frac{1}{2}} \sin ^{2 \lambda+1} \theta_{\nu, n}^{(\lambda)}\left[1-\frac{\lambda+2}{n+2 \lambda+2}\right]^{2}, \quad \lambda>-\frac{1}{2},
$$

while Winston [39], resp. Laden [26], have shown the monotonicity properties

$$
\begin{aligned}
& a_{\nu, n}^{(\lambda) G}<a_{\nu+1, n}^{(\lambda) G} \\
& a_{\nu, n}^{(\lambda) G} \sin ^{-4 \lambda} \theta_{\nu, n}^{(\lambda)}>a_{\nu+1, n}^{(\lambda) G} \sin ^{-4 \lambda} \theta_{\nu+1, n}^{(\lambda)} \text { for } \lambda>0, \nu<n / 2,
\end{aligned}
$$

and the validity of the reversed inequalities for $\lambda<0$. Note that all the above inequalities in (1.10) and (1.11) are asymptotically not sharp in the sense of (1.7). Inequalities for the first weight $a_{1, n}^{(\lambda) G}$ have been obtained by Bernstein [4] and Gatteschi and Vinardi [15]. Further explicit upper or lower bounds for all Gaussian weights $a_{\nu, n}^{(\lambda) G}$, with the exception of crude bounds (see, e.g., Monegato [27]), do not seem to be known.

The main purpose of this paper is to prove various simple but very accurate inequalities for the Gaussian weights $a_{\nu, n}^{(\lambda) G}$ involving only elementary functions. The upper and lower bounds obtained are asymptotically sharp, provided that $\nu$ increases as $n$ increases and $\lim _{\sup _{n \rightarrow \infty}} \nu / n<1$. Note that it follows from (1.9) that for fixed $\nu$ and increasing $n$, asymptotically sharp results are impossible without asymptotically sharp approximations of the zeros and values of Bessel functions. Furthermore, for nodes $x_{\nu, n}^{(\lambda) G}$ remaining in a fixed closed 
interval in the interior of $[-1,1]$, we derive inequalities and asymptotic values which are much sharper than those mentioned above.

\section{Statement of the Results}

Our first result improves the monotonicity properties (1.11) and makes them asymptotically correct in the sense of (1.7).

Theorem 1. Let $\nu<n / 2$; then, for the Gaussian weights $a_{\nu, n}^{(\lambda) G}$, if $\lambda \in[0,1]$,

$$
a_{\nu, n}^{(\lambda) G} \sin ^{-2 \lambda} \theta_{\nu, n}^{(\lambda)} \leq a_{\nu+1, n}^{(\lambda) G} \sin ^{-2 \lambda} \theta_{\nu+1, n}^{(\lambda)}
$$

where equality holds if and only if $\lambda \in\{0,1\}$. If $\lambda \notin(0,1)$, then the above result is valid if the inequality sign in (2.1) is reversed.

Remark 1. Complementing inequality $(2.1)$, we state that, for $\lambda \in[0,1]$ and $\nu<n / 2$,

$$
a_{\nu, n}^{(\lambda) G} \sin ^{-2 \lambda} \theta_{\nu, n}^{(\lambda)} \geq a_{\nu+1, n}^{(\lambda) G} \sin ^{-2 \lambda} \theta_{\nu+1, n}^{(\lambda)}\left(1-\rho_{\nu, n}^{(\lambda)}\right)^{1 / 2}
$$

where

$$
\rho_{\nu, n}^{(\lambda)}=\frac{\lambda(1-\lambda)}{(n+\lambda)^{2} \sin ^{2} \theta_{\nu, n}^{(\lambda)}+\lambda(1-\lambda)} \frac{\cos ^{2} \theta_{\nu, n}^{(\lambda)}-\cos ^{2} \theta_{\nu+1, n}^{(\lambda)}}{\sin ^{2} \theta_{\nu+1, n}^{(\lambda)}} .
$$

If $\lambda \notin(0,1)$, then this result is valid if the inequality sign is reversed, where for $\lambda<-4 / 11$ the inequality (2.2) for $\nu=1$ only holds if $(n+\lambda)^{2} \sin ^{2} \theta_{1, n}^{(\lambda)}>$ $\lambda(\lambda-1)$. Using $(2.3)$, we see that the monotonicity property $(2.1)$ is improvable only by a factor of the order $\left(1+\nu^{-3} O(1)\right)$ for $n \rightarrow \infty$ and $\nu \leq q n, q \in(0,1)$ fixed.

Remark 2. Note that it may be more helpful to consider the expression $\nu^{-k} O(1)$, where the $O$-estimate holds, as $n \rightarrow \infty$, uniformly for $\nu=1$, $2, \ldots,[q n]$, with $q$ fixed, $q \in(0,1)$, instead of $O\left(n^{-k}\right)$ as $n \rightarrow \infty$. If the nodes $x_{\nu, n}^{(\lambda) G}$ remain in a fixed closed interval lying in the interior of $[-1,1]$, then we have $O\left(n^{-k}\right)=\nu^{-k} O(1)$, but under the only assumption that $\nu$ increases, the term $\nu^{-k} O(1)$ additionally gives useful asymptotic results. Throughout this paper note that $\left[(n+\lambda) \sin \theta_{\nu, n}^{(\lambda)}\right]^{-1}=\nu^{-1} O(1)$ for every fixed $\lambda>-1 / 2$ and $\nu \leq q n, q \in(0,1)$ fixed, as $n \rightarrow \infty$, which follows from the known asymptotic approximation of $\theta_{\nu, n}^{(\lambda)}$ (see also (2.23)).

For odd $n, \lambda \in[0,1]$ and all $\nu$, one obtains from $(2.1)$ the inequality $a_{\nu, n}^{(\lambda) G} \leq a_{(n+1) / 2, n}^{(\lambda) G} \sin ^{2 \lambda} \theta_{\nu, n}^{(\lambda)}$ as well as the reversed inequality for $\lambda \notin$ $(0,1)$. Replacing $a_{(n+1) / 2, n}^{(\lambda)(i}$ by its representation (1.6), we obtain a simple upper bound, which, as a special case, is contained in the next theorem. Using 
the notations

$$
\begin{gathered}
c_{n}^{(\lambda)}:=\frac{\pi}{2} \frac{\Gamma\left(\frac{n}{2}+\frac{1}{2}\right) \Gamma\left(\frac{n}{2}+\lambda\right)}{\Gamma\left(\frac{n}{2}+1\right) \Gamma\left(\frac{n}{2}+\lambda+\frac{1}{2}\right)}, \\
c_{n}^{(\lambda) G}:= \begin{cases}c_{n}^{(\lambda)}=a_{(n+1) / 2, n}^{(\lambda) G} & \text { for odd } n, \\
c_{n-1}^{(\lambda+1)}\left(1-\lambda /\left((n+\lambda)^{2}+\lambda(1-\lambda)\right)\right) & \text { for even } n,\end{cases}
\end{gathered}
$$

we have the following inequalities, which are asymptotically sharp in the sense of (1.8).

Theorem 2. For the Gaussian weights $a_{\nu, n}^{(\lambda) G}$ we have, if $\lambda \in[0,1]$,

$$
a_{\nu, n}^{(\lambda) G}=c_{n}^{(\lambda) G} \sin ^{2 \lambda} \theta_{\nu, n}^{(\lambda)}\left(1+\varepsilon_{\nu, n}^{(\lambda)}\right),
$$

where

$$
-\frac{\lambda(1-\lambda) \cos ^{2} \theta_{\nu, n}^{(\lambda)}}{(n+\lambda)^{2} \sin ^{2} \theta_{\nu, n}^{(\lambda)}+\lambda(1-\lambda)} \leq \varepsilon_{\nu, n}^{(\lambda)} \leq 0 .
$$

Equality in (2.7) holds if and only if $\lambda \in\{0,1\}$ or $x_{\nu, n}^{(\lambda) G}=0$. For $\lambda \notin(0,1)$, the above results are also valid if the inequality signs in (2.7) are reversed, and where for $\lambda<-4 / 11$ the upper bound in (2.7) for $\nu=1$ or $\nu=n$ only holds if $(n+\lambda)^{2} \sin ^{2} \theta_{1, n}^{(\lambda)}>\lambda(\lambda-1)$.

Remark 3. For $\lambda>1$ the results of Theorem 2 can be improved as follows. Using

$$
\widetilde{c}_{n}^{(\lambda) G}:=\left\{\begin{array}{l}
\begin{array}{l}
c_{n}^{(\lambda)} \quad \text { for odd } n, \\
c_{n-1}^{(\lambda+1)}\left(1-\frac{\lambda}{(n+1)(n+2 \lambda-1)}-\frac{(2 \lambda-1)(\lambda-1)}{(n+1)^{2}(n+2 \lambda-1)^{2}}\right) \\
\text { for even } n
\end{array}
\end{array}\right.
$$

instead of $c_{n}^{(\lambda) G}$ in (2.6), we have that

$$
\begin{aligned}
& \frac{(\lambda-1)^{2} \cos ^{2} \theta_{\nu, n}^{(\lambda)}}{(n+\lambda)^{2} \sin ^{2} \theta_{\nu, n}^{(\lambda)}-(\lambda-1)(\lambda-2)} \\
& \leq \varepsilon_{\nu, n}^{(\lambda) G} \leq \frac{(\lambda-1) \cos ^{2} \theta_{\nu, n}^{(\lambda)}}{\left[(n+\lambda)^{2}-(\lambda-1)(\lambda-2)\right] \sin ^{2} \theta_{\nu, n}^{(\lambda)}}
\end{aligned}
$$

for $\lambda \in[1,2]$ and that the reversed inequalities hold for $\lambda \geq 2$. In particular, for $\lambda=2$, the upper and lower bound in (2.9) are equal, which gives the following simple representation for the weights $a_{\nu, n}^{(2) G}$ of the Gaussian formula $Q_{n}^{(2) G}$ :

$$
a_{\nu, n}^{(2) G}=\frac{\pi}{n+2} \sin ^{4} \theta_{\nu, n}^{(2)}\left\{1+\frac{1}{(n+1)(n+3) \sin ^{2} \theta_{\nu, n}^{(2)}}\right\} .
$$


For the nodes $x_{\nu, n}^{(2)}$, note that $\tan \left[(n+2) \theta_{\nu, n}^{(2)}\right]=(n+2) \tan \theta_{\nu, n}^{(2)}$.

The following inequalities yield an asymptotic approximation which is sharper than that given in (1.8). In particular, we have that for $\lambda \in[0,1]$, resp. $\lambda \notin(0,1)$, the geometric mean of the bounds (2.6) and (2.7) again is a lower, resp. upper, bound for the Gaussian weights.

Theorem 3. For the Gaussian weights $a_{\nu, n}^{(\lambda) G}$ we have, if $\lambda \in[0,1]$,

$$
\begin{aligned}
a_{\nu, n}^{(\lambda) G}= & c_{n}^{(\lambda) G} \sin ^{2 \lambda} \theta_{\nu, n}^{(\lambda)}\left\{1-\frac{\lambda(1-\lambda) \cos ^{2} \theta_{\nu, n}^{(\lambda)}}{(n+\lambda)^{2} \sin ^{2} \theta_{\nu, n}^{(\lambda)}+\lambda(1-\lambda)}\right\}^{1 / 2} \\
& \cdot\left\{1-\frac{\lambda(1-\lambda)\left[1+(-1)^{n}\right]}{4\left[(n+\lambda)^{2}+\lambda(1-\lambda)\right]^{2}}-\widetilde{\varepsilon}_{\nu, n}^{(\lambda)}\right\}^{-1},
\end{aligned}
$$

where

$$
0 \leq \widetilde{\varepsilon}_{\nu, n}^{(\lambda)} \leq \frac{\lambda(1-\lambda) \cos ^{2} \theta_{\nu, n}^{(\lambda)}\left[4-\cos ^{2} \theta_{\nu, n}^{(\lambda)}\right]}{2\left[(n+\lambda)^{2}+\lambda(1-\lambda)\right]^{2} \sin ^{4} \theta_{\nu, n}^{(\lambda)}} .
$$

For $\lambda \notin(0,1)$, the representation (2.11) is valid with

$$
\begin{aligned}
& -\frac{\lambda(\lambda-1) \cos ^{2} \theta_{\nu, n}^{(\lambda)}\left[4-\cos ^{2} \theta_{\nu, n}^{(\lambda)}\right]}{2\left[(n+\lambda)^{2} \sin ^{2} \theta_{\nu, n}^{(\lambda)}-\lambda(\lambda-1)\right]^{2}}\left\{1+\frac{\frac{3}{5} \lambda(\lambda-1)}{(n+\lambda)^{2} \sin ^{2} \theta_{\nu, n}^{(\lambda)}-\lambda(\lambda-1)}\right\}^{2} \\
& \quad \leq \widetilde{\varepsilon}_{\nu, n}^{(\lambda)} \leq 0
\end{aligned}
$$

where for $\lambda<-4 / 11$ the lower bound in (2.13) for $\nu=1$ or $\nu=n$ only holds if $(n+\lambda)^{2} \sin ^{2} \theta_{1, n}^{(\lambda)}>\lambda(\lambda-1)$. Equality in (2.12) or (2.13) is valid if and only if $\lambda \in\{0,1\}$ or $x_{\nu, n}^{(\lambda) G}=0$.

As a consequence of the above inequalities we have for fixed $\lambda$ and for $n \rightarrow \infty$

$$
\begin{array}{r}
a_{\nu, n}^{(\lambda) G}=\frac{\pi}{n+\lambda} \sin ^{2 \lambda} \theta_{\nu, n}^{(\lambda)}\left\{1-\frac{\lambda(1-\lambda)}{2(n+\lambda)^{2} \sin ^{2} \theta_{\nu, n}^{(\lambda)}}+\nu^{-4} O(1)\right\} \\
\nu \leq q n, q \in(0,1) \text { fixed. }
\end{array}
$$

For nodes $x_{\nu, n}^{(\hat{\lambda})}$ that remain in a fixed closed interval lying in the interior of $[-1,1]$ as $n \rightarrow \infty$, note that the asymptotic approximation (2.14) cannot be derived from (1.9).

In the above theorems we have used the quantity $c_{n}^{(\lambda) G}$ in order to obtain equality of our lower and upper bounds when $n$ is odd and $\nu=(n+1) / 2$. The following lemma shows that further simplifications are possible. 
Lemma 1. For fixed $\lambda>-1 / 2$ we have

$$
\begin{aligned}
c_{n}^{(\lambda)}=\frac{\pi}{n+\lambda}\left\{1+\frac{\lambda(\lambda-1)}{2}\right. & {\left[(n+\lambda)^{2}-(\lambda-1)^{2}\right.} \\
& \left.\left.+\frac{1}{4}(\lambda-2)(\lambda-3)\right]^{-1}+O\left(n^{-6}\right)\right\},
\end{aligned}
$$$$
n \rightarrow \infty \text {. }
$$

Furthermore, for every $n \in \mathbf{N}$, there exists $a \xi_{n}^{(\lambda)} \in\left[0, \frac{1}{4}\right]$ such that

$$
c_{n}^{(\lambda)}=\frac{\pi}{n+\lambda}\left\{1+\frac{\lambda(\lambda-1)}{2}\left[(n+\lambda)^{2}-(\lambda-1)^{2}+\xi_{n}^{(\lambda)}(\lambda-2)(\lambda-3)\right]^{-1}\right\} \text {. }
$$

In particular, we have, for all $n \in \mathbf{N}$,

$$
c_{n}^{(\lambda)}>\frac{\pi}{n+\lambda}\left\{1+\frac{\lambda(\lambda-1)}{2\left[(n+\lambda)^{2}+\lambda(1-\lambda)+\lambda / 4\right]}\right\} \quad \text { for } \lambda \in(0,1),
$$

and the reversed inequality $(2.17)$ if $\lambda \notin[0,1]$.

As a first consequence we obtain the following simple inequalities, which, in particular, show that for $\lambda \in(0,1)$ the asymptotic approximations $(1.7)$, resp. (2.14), are indeed upper, resp. lower, bounds.

Corollary 1. If $\lambda \in[0,1]$, then

$$
\frac{\pi}{n+\lambda} \sin ^{2 \lambda} \theta_{\nu, n}^{(\lambda)}\left\{1-\frac{\lambda(1-\lambda)}{2(n+\lambda)^{2} \sin ^{2} \theta_{\nu, n}^{(\lambda)}}\right\} \leq a_{\nu, n}^{(\lambda) G} \leq \frac{\pi}{n+\lambda} \sin ^{2 \lambda} \theta_{\nu, n}^{(\lambda)} .
$$

For $\lambda \notin(0,1)$,

$$
\begin{aligned}
& \frac{\pi}{n+\lambda} \sin ^{2 \lambda} \theta_{\nu, n}^{(\lambda)} \\
& \quad \leq a_{\nu, n}^{(\lambda) G} \leq \frac{\pi}{n+\lambda} \sin ^{2 \lambda} \theta_{\nu, n}^{(\lambda)}\left\{1+\frac{\lambda(\lambda-1)}{2(n+\lambda)^{2} \sin ^{2} \theta_{\nu, n}^{(\lambda)}-2 \lambda(\lambda-1)}\right\},
\end{aligned}
$$

where for $\lambda<-4 / 11$ the upper bound in (2.19) for $\nu=1$ or $\nu=n$ only holds if $(n+\lambda)^{2} \sin ^{2} \theta_{1, n}^{(\lambda)}>\lambda(\lambda-1)$. Equality in (2.18) and (2.19) holds if and only if $\lambda \in\{0,1\}$.

Using Theorems 1 and 2 and Lemma 1, various other simple inequalities can be obtained immediately. As an example, we state the following upper and lower bounds, which are both asymptotically sharp in the sense of (2.14).

Corollary 2. If $\lambda \in[0,1]$, then

$$
a_{\nu, n}^{(\lambda) G}=\frac{\pi}{n+\lambda} \sin ^{2 \lambda} \theta_{\nu, n}^{(\lambda)}\left\{1-\frac{\lambda(1-\lambda)}{(n+\lambda)^{2} \sin ^{2} \theta_{\nu, n}^{(\lambda)}}\right\}^{1 / 2}\left(1+\bar{\varepsilon}_{\nu, n}^{(\lambda)}\right),
$$

where

$$
0 \leq \bar{\varepsilon}_{\nu, n}^{(\lambda)} \leq \frac{2 \lambda(1-\lambda)}{(n+\lambda)^{4} \sin ^{4} \theta_{\nu, n}^{(\lambda)}}
$$


If $\lambda \geq 1$, the representation (2.20) holds with

$$
\begin{aligned}
& -\frac{\lambda(\lambda-1)(\lambda+1)^{3}}{4\left[(n+\lambda)^{2} \sin ^{2} \theta_{\nu, n}^{(\lambda)}-\lambda(\lambda-1)\right]^{2}} \\
& \quad \leq \bar{\varepsilon}_{\nu, n}^{(\lambda)} \leq \frac{3}{2} \frac{\lambda^{2}(\lambda-1)^{2}}{\left[(n+\lambda)^{2} \sin ^{2} \theta_{\nu, n}^{(\lambda)}-\lambda(\lambda-1)\right]^{2}} .
\end{aligned}
$$

Remark 4. For the trigonometric representation $\theta_{\nu, n}^{(\lambda)}$ of the node $x_{\nu, n}^{(\lambda) G}$ the following asymptotic approximation is valid for every fixed $\lambda>-\frac{1}{2}$ and $\nu \leq$ $q n, q \in(0,1)$ fixed, as $n \rightarrow \infty$ :

$$
\begin{array}{r}
\theta_{\nu, n}^{(\lambda)}=\phi_{\nu, n}^{(\lambda)}+\frac{\lambda(1-\lambda)}{2(n+\lambda)^{2}}\left\{1-\frac{6+\lambda(1-\lambda)\left[9-2 \cos ^{2} \phi_{\nu, n}^{(\lambda)}\right]}{\left.12(n+\lambda)^{2} \sin ^{2} \phi_{\nu, n}^{(\lambda)}\right]}\right. \\
\left.+\nu^{-4} O(1)\right\} \cot \phi_{\nu, n}^{(\lambda)},
\end{array}
$$

where

$$
\phi_{\nu, n}^{(\lambda)}:=\frac{2 \nu-1+\lambda}{n+\lambda} \frac{\pi}{2}
$$

(see Tricomi [37], Gatteschi [16], and Lemma 4 in $\S 3$ ). In particular, for $\lambda \in$ $[0,1]$ the following inequalities hold (Gatteschi [17]) for $\nu \leq(n+1) / 2$ :

$$
\phi_{\nu, n}^{(\lambda)} \leq \theta_{\nu, n}^{(\lambda)} \leq \phi_{\nu, n}^{(\lambda)}+\frac{\lambda(1-\lambda)}{2(n+\lambda)^{2}} \cot \phi_{\nu, n}^{(\lambda)} .
$$

Furthermore, if $\lambda \in[0,1]$ and $1<\nu \leq(n+1) / 2$ then, omitting the term $\nu^{-4} O(1)$ in (2.23), the right-hand side of (2.23) also is a lower bound for $\theta_{\nu, n}^{(\lambda)}$ [14]. Therefore, similar results as above, not requiring the explicit knowledge of the nodes $x_{\nu, n}^{(\lambda)}$, resp. $\theta_{\nu, n}^{(\lambda)}$, follow immediately. For the standard weight function $w \equiv 1$, i.e., $\lambda=\frac{1}{2}$, for example, we obtain that

$$
a_{\nu, n}^{(1 / 2) G}=\frac{2 \pi}{2 n+1} \sin \phi_{\nu, n}\left\{1-\frac{1}{2(2 n+1)^{2}}\right\}\left(1+\varepsilon_{\nu, n}\right),
$$

where

$$
\begin{gathered}
-\frac{\cos ^{2} \phi_{\nu, n}}{12\left(n+\frac{1}{2}\right)^{4} \sin ^{4} \phi_{\nu, n}}<\varepsilon_{\nu, n}<\frac{1}{2\left(n+\frac{1}{2}\right)^{4} \sin ^{4} \phi_{\nu, n}}, \\
\phi_{\nu, n}=\frac{4 \nu-1}{4 n+2} \pi .
\end{gathered}
$$

Finally, we state asymptotic approximations for the Gaussian weights which are sharper than $(2.14)$. 
Theorem 4. Let $\lambda>-\frac{1}{2}$; then, for $n \rightarrow \infty$ and $\nu \leq q n, q \in(0,1)$ fixed,

$$
\begin{gathered}
a_{\nu, n}^{(\lambda) G}=\frac{\pi}{n+\lambda} \sin ^{2 \lambda} \theta_{\nu, n}^{(\lambda)}\left\{1-\frac{\lambda(1-\lambda)}{(n+\lambda)^{2} \sin ^{2} \theta_{\nu, n}^{(\lambda)}+\lambda(1-\lambda)}\right\}^{1 / 2} \\
\cdot\left\{1+\frac{\lambda(1-\lambda)\left(1+2 \cos ^{2} \theta_{\nu, n}^{(\lambda)}\right)}{4(n+\lambda)^{4} \sin ^{4} \theta_{\nu, n}^{(\lambda)}}+\nu^{-6} O(1)\right\} \\
=\frac{\pi}{n+\lambda} \sin ^{2 \lambda} \phi_{\nu, n}^{(\lambda)}\left\{1-\frac{\lambda(1-\lambda)}{2(n+\lambda)^{2}}\left[1-(2 \lambda-1) \cot ^{2} \phi_{\nu, n}^{(\lambda)}\right]\right. \\
\left.+\frac{\lambda(1-\lambda) A^{(\lambda)}\left(\phi_{\nu, n}^{(\lambda)}\right)}{24(n+\lambda)^{4} \sin ^{4} \phi_{\nu, n}^{(\lambda)}}+\nu^{-6} O(1)\right\}, \\
A^{(\lambda)}(\theta):=9 \lambda(1-\lambda)+6+12(1-\lambda)\left(1+\lambda-3 \lambda^{2}\right) \cos ^{2} \theta \\
+4 \lambda^{2}(1-\lambda)(3 \lambda+1) \cos ^{4} \theta,
\end{gathered}
$$

where $\phi_{\nu, n}^{(\lambda)}$ is defined in (2.24). Furthermore,

$$
a_{\nu, n}^{(\lambda) G}=\frac{1}{2}\left(x_{\nu+1, n}^{(\lambda)}-x_{\nu-1, n}^{(\lambda)}\right) w_{\lambda}\left(x_{\nu, n}^{(\lambda)}\right)\left(\frac{\pi}{n+\lambda} \csc \frac{\pi}{n+\lambda}\right)\left(1+\nu^{-5} O(1)\right) .
$$

Note that (2.31) improves upon the trapezoidal theorem for Gaussian formulae of Davis and Rabinowitz [9, $§ 3$, equations (16) and (18)].

Remark 5. Consider the Lobatto quadrature formula $Q_{n}^{(\lambda) L o}$,

$$
Q_{n}^{(\lambda) L o}[f]=a_{1, n}^{(\lambda) L o} f(-1)+\sum_{\nu=2}^{n-1} a_{\nu, n}^{(\lambda) L o} f\left(x_{\nu, n}^{(\lambda) L o}\right)+a_{n, n}^{(\lambda) L o} f(1),
$$

defined uniquely by its having degree $2 n-3$ (see, e.g., Braß [5, p. 92]). Using the equations (cf. Krylov [24, pp. $160 \mathrm{ff}]$ )

$$
\begin{aligned}
& x_{\nu, n}^{(\lambda) L o}=x_{\nu-1, n-2}^{(\lambda+1) G}, \\
& a_{\nu, n}^{(\lambda) L o}=a_{\nu-1, n-2}^{(\lambda+1) G}\left[1-\left(x_{\nu-1, n-2}^{(\lambda+1) G}\right)^{2}\right]^{-1}, \quad \nu=2, \ldots, n-1,
\end{aligned}
$$

results similar to those above can be obtained immediately for the Lobatto formulae $Q_{n}^{(\lambda) L o}$. The values of $a_{1, n}^{(\lambda) L o}$, resp. $a_{n, n}^{(\lambda) L o}$, as well as the value of $a_{(n+1) / 2, n}^{(\lambda) L o}$ for odd $n$ are known explicitly (see, e.g., Ghizzetti and Ossicini [20, $\S 4.8])$. Note in Theorems 2 and 3 that $c_{n-1}^{(\lambda+1)}=a_{(n+1) / 2, n+1}^{(\lambda) L o}$ for odd $n$.

\section{Proof of the Results}

First, we state some preliminary lemmas on the zeros $x_{\nu, n}^{(\lambda)}$ of $P_{n}^{(\lambda)}$, which will be helpful for investigations near the boundary of the interval $[-1,1]$. For $\lambda \in\left(-\frac{1}{2}, 0\right)$ we require simple bounds for $x_{1, n}^{(\lambda)}$ following directly from results of Elbert [11]. 
Lemma 2. Let $\lambda \in\left(-\frac{1}{2}, 0\right]$ and $n>2$; then

$$
4\left[1+\frac{1}{5}\left(\lambda+\frac{1}{2}\right)\right]\left(\lambda+\frac{1}{2}\right)<(n+\lambda)^{2} \sin ^{2} \theta_{1, n}^{(\lambda)}<5\left(1+\frac{1}{3} \lambda\right)\left(\lambda+\frac{1}{2}\right) .
$$

Proof. By results of Szegö [35] and Buell [7] it is well known that

$$
\frac{j_{\lambda-1 / 2,1}}{n+\lambda}<\theta_{1, n}^{(\lambda)}<\frac{j_{\lambda-1 / 2,1}}{\left\{(n+\lambda)^{2}+\lambda(1-\lambda)\left[1-(2 / \pi)^{2}\right]\right\}^{1 / 2}} \quad \text { for } \lambda \notin[0,1],
$$

where as above, $j_{\lambda-1 / 2,1}$ denotes the smallest positive zero of the Bessel function $J_{\lambda-1 / 2}$, and that the reversed inequalities hold if $\lambda \in(0,1)$. Furthermore, Elbert [11] has proved that

$$
\begin{aligned}
2\left(\lambda+\frac{1}{2}\right)\left(\lambda+\frac{1}{2}+2\right) & \geq\left(j_{j-1 / 2,1}\right)^{2} \\
& \geq\left(\lambda+\frac{1}{2}\right)\left(\lambda+\frac{1}{2}+4\right) \quad \text { for } \lambda \in\left(-\frac{1}{2}, 0\right] .
\end{aligned}
$$

Substituting (3.3) in (3.2) and using the standard inequalities $0 \leq x^{2}-\sin ^{2} x \leq$ $x^{4} / 3,(3.1)$ follows for $n>5$. For $n=3,4,5$ a calculation of $1-\left(x_{\nu, n}^{(\lambda)}\right)^{2}$, using the explicit representation of the symmetric polynomials $P_{n}^{(\lambda)}$, proves (3.1).

For large $\lambda$ we need the following bound.

Lemma 3. Let $\lambda>-\frac{1}{2}$; then

$$
\left[(n+\lambda)^{2}-\frac{1}{4}\right] \sin ^{2} \theta_{1, n}^{(\lambda)} \geq\left(\lambda+\frac{1}{2}\right)^{2} .
$$

Note that for large $\lambda$ and small $n$ (in the sense of $n=o(\lambda)$ as $\lambda \rightarrow \infty$ ), Lemma 3 gives sharper estimates than those using the first zero of the Bessel function as in (3.2); in particular,

$$
\lim _{\lambda \rightarrow \infty} x_{\nu, n}^{(\lambda)}=0 \text { for } \lim _{\lambda \rightarrow \infty} n / \lambda=0 \text { and } \nu=1,2, \ldots, n \text {. }
$$

Since $j_{\lambda-1 / 2,1}=\left(\lambda-\frac{1}{2}\right)\left(1+O\left(\lambda^{-2 / 3}\right)\right)$ as $\lambda \rightarrow \infty$ (cf. Abramowitz and Stegun [1, equation 9.5.14]), the limit (3.5) cannot be derived from (3.2).

Proof of Lemma 3. We first note that the function $y_{n}^{(\lambda)}$ defined by

$$
y_{n}^{(\lambda)}(\theta):=(\sin \theta)^{\lambda-1 / 2} P_{n}^{(\lambda)}(\cos \theta), \quad \theta \in(0, \pi),
$$

is a solution of the differential equation

$$
y^{\prime \prime}+(\cot \theta) y^{\prime}+\left\{(n+\lambda)^{2}-\frac{1}{4}-\frac{\left(\lambda-\frac{1}{2}\right)^{2}}{\sin ^{2} \theta}\right\} y=0, \quad \theta \in(0, \pi) .
$$

This can be shown by explicit calculation, substituting $y_{n}^{(\lambda)}$ into (3.7) and using well-known identities for $P_{n}^{(\lambda)}$. Let $\alpha$ denote the first positive extremal point of $y_{n}^{(\lambda)}$ in $(0, \pi)$; then, for $\lambda>\frac{1}{2}$,

$$
y_{n}^{(\lambda)}(\alpha)>0, \quad y_{n}^{(\lambda)^{\prime}}(\alpha)=0, \quad y_{n}^{(\lambda)^{\prime \prime}}(\alpha)<0 \text {. }
$$


Therefore, (3.7) yields the inequality

$$
(n+\lambda)^{2}-\frac{1}{4}-\frac{\left(\lambda-\frac{1}{2}\right)^{2}}{\sin ^{2} \alpha}=-\frac{y_{n}^{(\lambda)^{\prime \prime}}(\alpha)}{y_{n}^{(\lambda)}(\alpha)}>0 .
$$

Now, let $\beta$ denote the smallest positive extremal point of $(\sin \theta)^{\lambda-1 / 2} y_{n}^{(\lambda)}(\theta)$. For $\lambda>\frac{1}{2}$ we have $\alpha \leq \beta$. By $-\cos \beta=x_{1, n+1}^{(\lambda-1)}=-\cos \theta_{1, n+1}^{(\lambda-1)}$ (cf. Abramowitz and Stegun $[1,22.13 .2])$ and (3.9) we obtain $\left[(n+\lambda)^{2}-\frac{1}{4}\right] \sin ^{2} \theta_{1, n+1}^{(\lambda-1)} \geq\left(\lambda-\frac{1}{2}\right)^{2}$ for $\lambda>\frac{1}{2}$, which proves the lemma.

Lemma 4. Let $i>-\frac{1}{2}$. Then, for $n \rightarrow \infty$ and $\nu \leq q n, q \in(0,1)$ fixed,

$$
\begin{array}{r}
\theta_{\nu, n}^{(\lambda)}=\phi_{\nu, n}^{(\lambda)}+\frac{\lambda(1-\lambda)}{2(n+\lambda)^{2}}\left\{1-\frac{6+\lambda(1-\lambda)\left[9-2 \cos ^{2} \phi_{\nu, n}^{(\lambda)}\right]}{12(n+\lambda)^{2} \sin ^{2} \phi_{\nu, n}^{(\lambda)}}\right. \\
\left.+\nu^{-4} O(1)\right\} \cot \phi_{\nu, n}^{(\lambda)},
\end{array}
$$

where

$$
\phi_{\nu, n}^{(\lambda)}:=\frac{2 \nu-1+\lambda}{n+\lambda} \frac{\pi}{2}
$$

For nodes $x_{\nu, n}^{(\lambda) G}$ remaining in a fixed closed interval in the interior of $[-1,1]$, the above asymptotic approximation follows immediately from results of Tricomi [37] and Gatteschi [16].

Proof of Lemma 4. We first prove for every fixed $\lambda>-\frac{1}{2}$ the much weaker approximation $\theta_{\nu, n}^{(\lambda)}=\phi_{\nu, n}^{(\lambda)}+\lambda(1-\lambda)\left[2(n+\lambda)^{2}\right]^{-1}\left[1+O\left(n^{-1} \sin ^{-1} \theta_{\nu, n}^{(\lambda)}\right] \cot \phi_{\nu, n}^{(\lambda)}\right.$ by use of Sturm's comparison theorem applied to

$$
\begin{gathered}
u_{n}^{(\lambda)^{\prime \prime}}+g_{n}^{(\lambda)} u_{n}^{(\lambda)}=0, \quad g_{n}^{(\lambda)}(\theta)=(n+\lambda)^{2}+\frac{\lambda(1-\lambda)}{\sin ^{2} \theta}, \\
u_{n}^{(\lambda)}(\theta)=\sin ^{\lambda} \theta P_{n}^{(\lambda)}(\cos \theta)
\end{gathered}
$$

(cf. Szegö [36, equation (4.7.11)]) and using symmetry of $P_{n}^{(\lambda)}$. Then a repeated application of the asymptotic approximation

$$
\begin{aligned}
& \frac{\Gamma(\lambda) \Gamma(n+\lambda+1) \sin ^{\lambda} \theta}{2 \Gamma(n+2 \lambda)} P_{n}^{(\lambda)}(\cos \theta) \\
& \quad=\sum_{\nu=0}^{p} \frac{b_{\nu}}{\sin ^{\nu} \theta} \cos \left[(n+\lambda+\nu) \theta-(\lambda+\nu) \frac{\pi}{2}\right]+M b_{p+1} \frac{1}{\sin ^{p+1} \theta}, \\
& |M| \leq \frac{2^{p+1}}{\sin \lambda \pi}, \quad b_{0}=1, \quad b_{\nu}=\frac{1}{\nu ! 2^{\nu}} \prod_{\mu=1}^{\prime \prime} \frac{(\mu-\lambda)(\mu+\lambda-1)}{n+\lambda+\mu} \text { for } \nu>0,
\end{aligned}
$$

proved by Szegö [34] for $p>\lambda-1$ and nonintegral $\lambda$, yields (3.10). For integral $\lambda$ one can use the representation given in Szegö [36, equation (8.4.13)]. The calculations are lengthy but straightforward. 
Lemma 5. Let $\lambda>-\frac{1}{2}$. Then, for $n \rightarrow \infty$ and $2 \leq \nu \leq q n, q \in(0,1)$ fixed,

$$
\begin{aligned}
x_{\nu+1, n}^{(\lambda)}-x_{\nu-1, n}^{(\lambda)}= & \frac{2 \pi}{n+\lambda}\left[1-\left(x_{\nu, n}^{(\lambda)}\right)^{2}\right]^{1 / 2} \\
& \cdot\left\{1-\frac{B^{(\lambda)}\left(x_{\nu, n}^{(\lambda)}\right)}{2(n+\lambda)^{2}}+\frac{C^{(\lambda)}\left(x_{\nu, n}^{(\lambda)}\right)}{24(n+\lambda)^{4}}+\nu^{-5} O(1)\right\},
\end{aligned}
$$

where

$$
\begin{gathered}
B^{(\lambda)}(x):=\frac{\pi^{2}}{3}+\frac{\lambda(1-\lambda)}{1-x^{2}} \\
C^{(\lambda)}(x):=\frac{\pi^{4}}{5}+\left(2 \pi^{2}-12\right) \frac{\lambda(1-\lambda)}{1-x^{2}}+9 \frac{(1+\lambda) \lambda(1-\lambda)(2-\lambda)}{\left(1-x^{2}\right)^{2}}
\end{gathered}
$$

Proof. Using Taylor's expansion in (3.10), we obtain

$$
\begin{aligned}
\theta_{\nu+1, n}^{(\lambda)}-\theta_{\nu, n}^{(\lambda)} & =\frac{\pi}{n+\lambda}\left\{1+\frac{\lambda(1-\lambda) \pi \cos \theta_{\nu, n}^{(\lambda)}}{2(n+\lambda)^{3} \sin ^{3} \theta_{\nu, n}^{(\lambda)}}-\lambda(1-\lambda) H_{n}^{(\lambda)}\left(\theta_{\nu, n}^{(\lambda)}\right)+\nu^{-5} O(1)\right\} \\
= & \frac{\pi}{n+\lambda}\left\{1-\frac{\lambda(1-\lambda) \pi \cos \theta_{\nu+1, n}^{(\lambda)}}{2(n+\lambda)^{3} \sin ^{3} \theta_{\nu+1, n}^{(\lambda)}}-\lambda(1-\lambda) H_{n}^{(\lambda)}\left(\theta_{\nu+1, n}^{(\lambda)}\right)+\nu^{-5} O(1)\right\} \\
= & \frac{\pi}{n+\lambda}\left\{1+\frac{\lambda(1-\lambda)}{(n+\lambda)^{2} \sin ^{2} \bar{\theta}_{\nu, n}^{(\lambda)}}\left[1+\frac{\left(\pi^{2}-6\right)\left(1+2 \cos ^{2} \bar{\theta}_{\nu, n}^{(\lambda)}\right)}{12(n+\lambda)^{2} \sin ^{2} \bar{\theta}_{\nu, n}^{(\lambda)}}\right]\right\}^{-1 / 2} \\
& \cdot\left(1+\nu^{-5} O(1)\right),
\end{aligned}
$$

where

$$
\begin{gathered}
H_{n}^{(\lambda)}(\theta):=\frac{1}{2(n+\lambda)^{2} \sin ^{2} \theta}+\frac{\left(4 \pi^{2}-6\right)\left(1+2 \cos ^{2} \theta\right)-9 \lambda(1-\lambda)}{24(n+\lambda)^{4} \sin ^{4} \theta}, \\
\bar{\theta}_{\nu, n}^{(\lambda)}:=\left(\theta_{\nu, n}^{(\lambda)}+\theta_{\nu+1, n}^{(\lambda)}\right) / 2 .
\end{gathered}
$$

The assertion follows by noting $x_{\nu, n}^{(\lambda)}=-\cos \theta_{\nu, n}^{(\lambda)}$ and again applying Taylor's expansion.

Proof of Lemma 1. For fixed $\xi$ and $\lambda$ we consider the sequence

$$
d_{n, \xi}^{(\lambda)}:=c_{n}^{(\lambda)} \frac{n+\lambda}{\pi}\left\{1+\frac{\lambda(\lambda-1)}{2}[n(n+2 \lambda)+\xi]^{-1}\right\}^{-1} .
$$

Using known asymptotic approximations for the gamma function (cf., e.g., Erdélyi and Tricomi [12]), we have (2.15) and the much weaker relation

$$
\lim _{n \rightarrow \infty} d_{n, \xi}^{(\lambda)}=1 \text {. }
$$


A somewhat lengthy but straightforward calculation using the recurrence formula of the gamma function shows that

$$
d_{n, \xi}^{(\lambda)} \geq d_{n+2, \xi}^{(\lambda)}
$$

holds if and only if

$$
\begin{aligned}
\lambda(\lambda-1)\{( & \left.4 \xi-\lambda^{2}-3 \lambda-2\right)\left[n^{2}+2(\lambda+1) n\right] \\
& \left.+2 \xi^{2}+\xi\left(\lambda^{2}+7 \lambda+8\right)-4 \lambda(\lambda+1)(\lambda+2)\right\} \geq 0 .
\end{aligned}
$$

If $4 \xi:=(\lambda+2)(\lambda+1)$, then the last inequality is equivalent to

$$
(\lambda+2)(\lambda+1) \lambda(\lambda-1)(\lambda-2)(\lambda-3) \geq 0 .
$$

If $\xi=2 \lambda-1$, then (3.22) is equivalent to

$$
\lambda(\lambda-1)(\lambda-2)(\lambda-3)\left[n^{2}+2(\lambda+1)(n+1)-1\right] \leq 0 .
$$

Substituting these two special values for $\xi$ in (3.19) and using (3.20) and (3.21), the assertion (2.16) follows. Using the same method for $\xi=\frac{5}{4} \lambda$, we obtain (2.17).

Proof of Theorems 1-4. We consider the following representation of the Gaussian weights $a_{\nu, n}^{(\lambda) G}$ which follows from (1.5):

$$
\begin{aligned}
a_{\nu, n}^{(\lambda) G}= & c_{n}^{(\lambda) G} \sin ^{2 \lambda} \theta_{\nu, n}^{(\lambda)}\left\{v_{1, n}^{(\lambda)}\left(\theta_{\nu, n}^{(\lambda)}\right)\right\}^{-1} \\
= & c_{n}^{(\lambda) G} \sin ^{2 \lambda} \theta_{\nu, n}^{(\lambda)}\left\{1-\frac{\lambda(1-\lambda) \cos ^{2} \theta_{\nu, n}^{(\lambda)}}{(n+\lambda)^{2} \sin ^{2} \theta_{\nu, n}^{(\lambda)}+\lambda(1-\lambda)}\right\} \\
& \cdot\left\{v_{2, n}^{(\lambda)}\left(\theta_{\nu, n}^{(\lambda)}\right)\right\}^{-1}
\end{aligned}
$$

with $c_{n}^{(\lambda) G}$ defined in (2.5) and

$$
v_{1, n}^{(\lambda)}(\theta)=\gamma_{n}^{(\lambda)} \sin ^{2 \lambda} \theta\left\{\left[\sin \theta P_{n}^{(\lambda)^{\prime}}(\cos \theta)\right]^{2}-2 \lambda \cos \theta P_{n}^{(\lambda)}(\cos \theta) P_{n}^{(\lambda)^{\prime}}(\cos \theta)\right.
$$

$$
\begin{array}{r}
\left.+\left((n+\lambda)^{2}+\lambda(1-\lambda)+\lambda \cot ^{2} \theta\right)\left[P_{n}^{(\lambda)}(\cos \theta)\right]^{2}\right\}, \\
\theta \in(0, \pi),
\end{array}
$$

$$
\begin{aligned}
& v_{2, n}^{(\lambda)}(\theta)=v_{1, n}^{(\lambda)}(\theta)\left\{1-\frac{\lambda(1-\lambda) \cos ^{2} \theta}{(n+\lambda)^{2} \sin ^{2} \theta+\lambda(1-\lambda)}\right\}, \\
& \theta \in(\bar{\theta}, \pi-\bar{\theta}),
\end{aligned}
$$

where

$$
\begin{aligned}
& \gamma_{n}^{(\lambda)}=c_{n}^{(\lambda) G} \frac{[\Gamma(\lambda)]^{2} \Gamma(n+1)}{2^{2-2 \lambda} \pi \Gamma(n+2 \lambda)}, \\
& \sin ^{2} \bar{\theta}:=\max \left\{0, \frac{\lambda(\lambda-1)}{(n+\lambda)^{2}}\right\}, \quad \bar{\theta} \in\left[0, \frac{\pi}{2}\right] .
\end{aligned}
$$


Using Lemmas 2 and 3, it follows that

$$
\theta_{1, n}^{(\lambda)}>\bar{\theta} \quad \text { for } n>2 \text { and } \lambda \geq(29-\sqrt{885}) / 2=-0.37447 \ldots
$$

while

$$
\theta_{1, n}^{(\lambda)}<\bar{\theta} \text { for } n>2 \text { and } \lambda \leq(\sqrt{1441}-41) / 8=-0.37993 \ldots
$$

For $n=2$, we have by explicit calculation of $x_{1,2}^{(\lambda)}$ that $\theta_{1,2}^{(\lambda)} \geq \bar{\theta}$ if and only if $\lambda \geq(\sqrt{13}-7) / 9=-0.37716 \ldots$. Note that from the inequalities obtained by Buell [7, p. 311] one has $\theta_{2, n}^{(\hat{\lambda})}>\bar{\theta}$ for all $\lambda>-\frac{1}{2}$. Furthermore, standard identities for $P_{n}^{(\lambda)}$ (cf. Szegö [36, §4.7] ) yield

$$
P_{n}^{(\lambda)}\left(\cos _{\nu, n-1}^{(\lambda+1)}\right)=-\frac{2 \lambda}{n(n+2 \lambda)} \sin ^{2} \theta_{\nu, n-1}^{(\lambda+1)} P_{n-1}^{(\lambda+1)^{\prime}}\left(\cos \theta_{\nu, n-1}^{(\lambda+1)}\right)
$$

and hence, for $\lambda>-\frac{1}{2}$,

$$
a_{\nu, n-1}^{(\lambda+1) G}=\widetilde{c}_{n-1}^{(\lambda+1) G} \sin ^{2 \lambda+2} \theta_{\nu, n-1}^{(\lambda+1)}\left\{1+\frac{\lambda \cos ^{2} \theta_{\nu, n-1}^{(\lambda+1)}}{\left[(n+\lambda)^{2}+\lambda(1-\lambda)\right] \sin ^{2} \theta_{\nu, n-1}^{(\lambda+1)}}\right\}
$$

$$
\begin{aligned}
& \cdot\left(v_{1, n}^{(\lambda)}\left(\theta_{\nu, n-1}^{(\lambda+1)}\right)\right)^{-1} \\
= & \widetilde{c}_{n-1}^{(\lambda+1) G} \sin ^{2 \lambda+2} \theta_{\nu, n-1}^{(\lambda+1)}\left\{1+\frac{\lambda^{2} \cos ^{2} \theta_{\nu, n-1}^{(\lambda+1)}}{(n+\lambda)^{2} \sin ^{2} \theta_{\nu, n-1}^{(\lambda+1)}+\lambda(1-\lambda)}\right\} \\
& \cdot\left(v_{2, n}^{(\lambda)}\left(\theta_{\nu, n-1}^{(\lambda+1)}\right)\right)^{-1}
\end{aligned}
$$

with $\widetilde{c}_{n-1}^{(\lambda+1)}$ defined in (2.8). An explicit calculation gives

$$
\begin{aligned}
v_{1, n}^{(\lambda)}\left(\frac{\pi}{2}\right) & =v_{2, n}^{(\lambda)}\left(\frac{\pi}{2}\right) \\
& =\gamma_{n}^{(\lambda)} \sin ^{2 \lambda} \theta\left\{\left[(n+\lambda)^{2}+\lambda(1-\lambda)\right]\left[P_{n}^{(\lambda)}(0)\right]^{2}+\left[P_{n}^{(\lambda)^{\prime}}(0)\right]^{2}\right\} \\
& =1 .
\end{aligned}
$$

Therefore, to prove Theorem 2 as well as Remark 3, it suffices to show that

$$
\begin{gathered}
v_{1, n}^{(\lambda)}(\theta)\left\{\begin{array}{l}
\geq v_{1, n}^{(\lambda)}(\pi / 2) \text { for } \lambda \in[0,1], \\
\leq v_{1, n}^{(\lambda)}(\pi / 2) \text { for } \lambda \notin[0,1],
\end{array}\right. \\
v_{2, n}^{(\lambda)}(\theta)\left\{\begin{array}{l}
\leq v_{2, n}^{(\lambda)}(\pi / 2) \\
\geq v_{2, n}^{(\lambda)}(\pi / 2) \text { for } \lambda \in[0,1],
\end{array} \quad \theta \in(0, \pi)\right.
\end{gathered}
$$

and that equality in (3.34) and (3.35) holds if and only if $\lambda \in\{0,1\}$ or $\theta=$ $\pi / 2$. To verify this, we apply standard methods introduced by Szegö $[36, \S 7.3]$ and Bernstein [4]. First note that an explicit calculation yields, in the notation of (3.12),

$$
v_{1, n}^{(\lambda)}(\theta)=\gamma_{n}^{(\lambda)}\left\{g_{n}^{(\lambda)}(\theta)\left[u_{n}^{(\lambda)}(\theta)\right]^{2}+\left[u_{n}^{(\lambda)^{\prime}}(\theta)\right]^{2}\right\}
$$


It follows that

$$
v_{1, n}^{(\lambda)^{\prime}}(\theta)=\gamma_{n}^{(\lambda)}\left[u_{n}^{(\lambda)}(\theta)\right]^{2} g_{n}^{(\lambda)^{\prime}}(\theta) .
$$

The function $g_{n}^{(\lambda)}$ is monotonic in $(0, \pi / 2)$, resp. $(\pi / 2, \pi)$, and therefore (3.34) holds. Similarly, we have

$$
v_{2, n}^{(\lambda)}(\theta)=\frac{(n+\lambda)^{2}+\lambda(1-\lambda)}{g_{n}^{(\lambda)}(\theta)} v_{1, n}^{(\lambda)}(\theta), \quad \theta \in(\bar{\theta}, \pi-\bar{\theta}),
$$

and (cf. Szegö [36, §7.31])

$$
v_{2, n}^{(\lambda)^{\prime}}(\theta)=-\gamma_{n}^{(\lambda)}\left[(n+\lambda)^{2}+\lambda(1-\lambda)\right]\left[\frac{u_{n}^{(\lambda)^{\prime}}(\theta)}{g_{n}^{(\lambda)}(\theta)}\right]^{2} g_{n}^{(\lambda)^{\prime}}(\theta),
$$

$$
\theta \in(\bar{\theta}, \pi-\bar{\theta})
$$

This gives (3.35).

By (3.37) and (3.39) the functions $v_{1, n}^{(\lambda)}$ and $v_{2, n}^{(\lambda)}$ are monotonic in $(0, \pi / 2)$. Therefore, Theorem 1 is a direct consequence of the representations (3.25), resp. (3.26).

To prove Theorem 3 , we consider the following representation of the weights

$$
a_{\nu, n}^{(\lambda) G}=c_{n}^{(\lambda) G} \frac{\sin ^{2 \lambda} \theta_{\nu, n}^{(\lambda)}}{v_{3, n}^{(\lambda)}\left(\theta_{\nu, n}^{(\lambda)}\right)}\left\{\frac{(n+\lambda)^{2}+\lambda(1-\lambda)}{g_{n}^{(\lambda)}\left(\theta_{\nu, n}^{(\lambda)}\right)}\right\}^{1 / 2},
$$

where the function $v_{3, n}^{(\lambda)}$ is defined by

$$
\begin{aligned}
v_{3, n}^{(\lambda)}(\theta)= & \gamma_{n}^{(\lambda)}\left\{\frac{(n+\lambda)^{2}+\lambda(1-\lambda)}{g_{n}^{(\lambda)}(\theta)}\right\}^{1 / 2} \\
& \cdot\left\{\left(g_{n}^{(\lambda)}(\theta)-\frac{g_{n}^{(\lambda)^{\prime \prime}}(\theta)}{4 g_{n}^{(\lambda)}(\theta)}+\frac{3\left[g_{n}^{(\lambda)^{\prime}}(\theta)\right]^{2}}{8\left[g_{n}^{(\lambda)}(\theta)\right]^{2}}\right)\left[u_{n}^{(\lambda)}(\theta)\right]^{2}\right. \\
& \left.+\left[u_{n}^{(\lambda)^{\prime}}(\theta)\right]^{2}+\frac{g_{n}^{(\lambda)^{\prime}}(\theta)}{2 g_{n}^{(\lambda)}(\theta)} u_{n}^{(\lambda)}(\theta) u_{n}^{(\lambda)^{\prime}}(\theta)\right\}
\end{aligned}
$$

for $\theta \in(\bar{\theta}, \pi-\bar{\theta})$. Using (3.12), we obtain

$$
\begin{aligned}
v_{3, n}^{(\lambda)^{\prime}}(\theta)= & -\gamma_{n}^{(\lambda)}\left[(n+\lambda)^{2}+\lambda(1-\lambda)\right]^{1 / 2} \frac{\left[u_{n}^{(\lambda)}(\theta)\right]^{2}}{4} \\
& \cdot\left\{g_{n}^{(\lambda))^{\prime}}(\theta)\left[g_{n}^{(\lambda)}(\theta)\right]^{-3 / 2}\right\}^{\prime \prime} \\
= & -\gamma_{n}^{(\lambda)}\left[(n+\lambda)^{2}+\lambda(1-\lambda)\right]^{1 / 2} \frac{\left[u_{n}^{(\lambda)}(\theta)\right]^{2} g_{n}^{(\lambda)^{\prime \prime \prime}}(\theta)}{4\left[g_{n}^{(\lambda)}(\theta)\right]^{7 / 2}} \\
& \cdot\left\{\left[g_{n}^{(\lambda)}(\theta)\right]^{2}-\frac{9}{2} \frac{g_{n}^{(\lambda)^{\prime \prime}}(\theta) g_{n}^{(\lambda)^{\prime}}(\theta)}{g_{n}^{(\lambda)^{\prime \prime \prime}}(\theta)} g_{n}^{(\lambda)}(\theta)+\frac{15}{4} \frac{\left[g_{n}^{(\lambda)^{\prime}}(\theta)\right]^{3}}{g_{n}^{(\lambda)^{\prime \prime \prime}}(\theta)}\right\} .
\end{aligned}
$$


Furthermore, we have

$$
\begin{aligned}
v_{3, n}^{(\lambda)}(\theta) & =v_{3, n}^{(\lambda)}(\pi / 2)-\int_{\theta}^{\pi / 2} v_{3, n}^{(\lambda)^{\prime}}(t) d t \\
& =1-\frac{\gamma_{n}^{(\lambda)}}{4\left[(n+\lambda)^{2}+\lambda(1-\lambda)\right]}\left[u_{n}^{(\lambda)}(\pi / 2)\right]^{2} g_{n}^{(\lambda)^{\prime \prime}}(\pi / 2)-\widetilde{\varepsilon}_{\nu, n}^{(\lambda)} \\
& =1-\frac{\lambda(1-\lambda)\left[1+(-1)^{n}\right]}{4\left[(n+\lambda)^{2}+\lambda(1-\lambda)\right]^{2}}-\widetilde{\varepsilon}_{\nu, n}^{(\lambda)},
\end{aligned}
$$

where

$$
\begin{aligned}
\widetilde{\varepsilon}_{\nu, n}^{(\lambda)} & =\int_{\theta}^{\pi / 2} v_{3, n}^{(\lambda)^{\prime}}(t) d t \\
& =-\frac{\gamma_{n}^{(\lambda)}}{4}\left[(n+\lambda)^{2}+\lambda(1-\lambda)\right]^{1 / 2} \int_{\theta}^{\pi / 2}\left[u_{n}^{(\lambda)}(t)\right]^{2} \cdot\left(\frac{g_{n}^{(\lambda)}(t)}{\left[g_{n}^{(\lambda)}(t)\right]^{3 / 2}}\right)^{\prime \prime} d t .
\end{aligned}
$$

First let $\lambda \in(0,1)$. The term is curly brackets in $(3.43)$ is positive if

$$
g_{n}^{(\lambda)}(\theta)>\frac{9}{2} \frac{g_{n}^{(\lambda)^{\prime \prime}}(\theta) g_{n}^{(\lambda)^{\prime}}(\theta)}{g_{n}^{(\lambda)^{\prime \prime \prime}}(\theta)}=\frac{9}{4} \frac{\lambda(1-\lambda)}{\sin ^{2} \theta} \frac{1+2 \cos ^{2} \theta}{2+\cos ^{2} \theta},
$$

and therefore if

$$
(n+\lambda)^{2}>\frac{5}{4} \frac{\lambda(1-\lambda)}{\sin ^{2} \theta} .
$$

For $\theta=\theta_{1, n}^{(\lambda)}$, this inequality follows from Lemma 3, so that the lower bound in (2.12) is proved. Since the integrand of the last integral in (3.45) is negative, we estimate $\left[u_{n}^{(\lambda)}(t)\right]^{2}$ by $\left(\gamma_{n}^{(\lambda)}\left[(n+\lambda)^{2}+\lambda(1-\lambda)\right]\right)^{-1}(\mathrm{cf} .(3.35),(3.36)$, and (3.38)). Now, an explicit calculation of the resulting integral yields

$$
\begin{gathered}
\widetilde{\varepsilon}_{\nu, n}^{(\lambda)} \leq \frac{\lambda(1-\lambda)}{2} \frac{\left(1+2 \cos ^{2} \theta_{\nu, n}^{(\lambda)}-3 h_{\nu, n}^{(\lambda)}\right)\left(1-h_{\nu, n}^{(\lambda)}\right)^{3 / 2}-\sin ^{4} \theta_{\nu, n}^{(\lambda)}}{\left[(n+\lambda)^{2}+\lambda(1-\lambda)\right]^{2} \sin ^{4} \theta_{\nu, n}^{(\lambda)}} \\
h_{\nu, n}^{(\lambda)}:=\frac{\lambda(1-\lambda) \cos ^{2} \theta_{\nu, n}^{(\lambda)}}{(n+\lambda)^{2} \sin ^{2} \theta_{\nu, n}^{(\lambda)}+\lambda(1-\lambda)} .
\end{gathered}
$$

A further estimation proves the upper bound in (2.12). For $\lambda \notin[0,1]$ and $\theta_{\nu, n}^{(\lambda)} \in(\bar{\theta}, \pi / 2]$, the upper bound in (2.13) follows from the positivity of each summand in the curly brackets in (3.43). To prove the lower bound in (2.13), we notice that $\left[u_{n}^{(\lambda)}(t)\right]^{2}$ can be estimated from above by $\left[\gamma_{n}^{(\lambda)} g_{n}^{(\lambda)}(t)\right]^{-1}$ (cf. 
(3.34) and (3.36)). Therefore, we have for $\lambda \notin[0,1]$ and $\theta_{\nu, n}^{(\lambda)} \in(\bar{\theta}, \pi-\bar{\theta})$

$$
\begin{aligned}
\widetilde{\varepsilon}_{\nu, n}^{(\lambda)} \geq & -\frac{\left[(n+\lambda)^{2}+\lambda(1-\lambda)\right]^{1 / 2}}{4} \\
& \cdot \int_{\theta}^{\pi / 2} \frac{g_{n}^{(\lambda)^{\prime \prime \prime}}(t)}{\left[g_{n}^{(\lambda)}(t)\right]^{5 / 2}}-\frac{9 g_{n}^{(\lambda)^{\prime}}(t) g_{n}^{(\lambda)^{\prime \prime}}(t)}{2\left[g_{n}^{(\lambda)}(t)\right]^{7 / 2}}+\frac{15\left[g_{n}^{(\lambda)^{\prime}}(t)\right]^{3}}{4\left[g_{n}^{(\lambda)}(t)\right]^{9 / 2}} d t \\
= & -\frac{\left[(n+\lambda)^{2}+\lambda(1-\lambda)\right]^{1 / 2}}{4} \\
& \cdot\left[\frac{32}{35 \lambda(\lambda-1)} \frac{1}{\left[g_{n}^{(\lambda)}(t)\right]^{1 / 2}}-\frac{16}{105} \frac{2+\cos ^{2} t}{\sin ^{2} t\left[g_{n}^{(\lambda)}(t)\right]^{3 / 2}}\right. \\
& \left.+\frac{33}{35} \frac{g_{n}^{(\lambda)^{\prime \prime}}(t)}{\left[g_{n}^{(\lambda)}(t)\right]^{5 / 2}}-\frac{15}{14} \frac{\left[g_{n}^{(\lambda)^{\prime}}(t)\right]^{2}}{\left[g_{n}^{(\lambda)}(t)\right]^{7 / 2}}\right]_{\theta}^{\pi / 2} .
\end{aligned}
$$

By explicit calculation we now obtain a lower bound for $\widetilde{\varepsilon}_{\nu, n}^{(\lambda)}$ which is larger than the left-hand side of (2.13).

For the proof of Theorem 4 we use the auxiliary function

$$
\begin{aligned}
v_{4, n}^{(\lambda)}(\theta)= & v_{3, n}^{(\lambda)}(\theta)+\frac{1}{8\left[(n+\lambda)^{2}+\lambda(1-\lambda)\right]^{1 / 2}}\left(\frac{g_{n}^{(\lambda)^{\prime}}(\theta)}{\left[g_{n}^{(\lambda)}(\theta)\right]^{3 / 2}}\right)^{\prime} v_{2, n}^{(\lambda)}(\theta) \\
(3.52) \quad & -\frac{\gamma_{n}^{(\lambda)}\left[(n+\lambda)^{2}+\lambda(1-\lambda)\right]^{1 / 2}}{8 g_{n}^{(\lambda)}}\left(\frac{g_{n}^{(\lambda)}(\theta)}{\left[g_{n}^{(\lambda)}(\theta)\right]^{3 / 2}}\right)^{\prime \prime} u_{n}^{(\lambda)}(\theta) u_{n}^{(\lambda)^{\prime}}(\theta) \\
& +\frac{\gamma_{n}^{(\lambda)}\left[(n+\lambda)^{2}+\lambda(1-\lambda)\right]^{1 / 2}}{16}\left\{\frac{1}{g_{n}^{(\lambda)}(\theta)}\left(\frac{g_{n}^{(\lambda)}(\theta)}{\left[g_{n}^{(\lambda)}(\theta)\right]^{3 / 2}}\right)^{\prime \prime}\right\}^{\prime}\left[u_{n}^{(\lambda)}(\theta)\right]^{2} .
\end{aligned}
$$

Its derivative is

$$
\begin{aligned}
v_{4, n}^{(\lambda)^{\prime}}(\theta)= & \frac{\gamma_{n}^{(\lambda)}\left[(n+\lambda)^{2}+\lambda(1-\lambda)\right]^{1 / 2}}{16}\left\{\frac{1}{g_{n}^{(\lambda)}(\theta)}\left(\frac{g_{n}^{(\lambda)^{\prime}}(\theta)}{\left[g_{n}^{(\lambda)}(\theta)\right]^{3 / 2}}\right)^{\prime \prime}\right\}^{\prime \prime}\left[u_{n}^{(\lambda)}(\theta)\right]^{2} \\
3.53) \quad & -\frac{\gamma_{n}^{(\lambda)}\left[(n+\lambda)^{2}+\lambda(1-\lambda)\right]^{1 / 2}}{8} \frac{g_{n}^{(\lambda)^{\prime}}(\theta)}{\left[g_{n}^{(\lambda)}(\theta)\right]^{2}}\left(\frac{g_{n}^{(\lambda)^{\prime}}(\theta)}{\left[g_{n}^{(\lambda)}(\theta)\right]^{3 / 2}}\right)^{\prime}\left[u_{n}^{(\lambda)^{\prime}}(\theta)\right]^{2} \\
= & O\left(n^{-6} \sin ^{-7} \theta\right) .
\end{aligned}
$$

Therefore, the function $v_{4, n}^{(i)}(\theta)$ differs from $v_{4, n}^{(\hat{\lambda})}(\pi / 2)$ only by a term of order 
$O\left(n^{-6} \sin ^{-6} \theta\right)$, so that

$$
a_{\nu, n}^{(\lambda) G}=c_{n}^{(\lambda) G} \frac{\sin ^{2 \lambda} \theta_{\nu, n}^{(\lambda)}}{v_{4, n}\left(\theta_{\nu, n}^{(\lambda)}\right)}\left\{\frac{(n+\lambda)^{2}+\lambda(1-\lambda)}{g_{n}^{(\lambda)}\left(\theta_{\nu, n}^{(\lambda)}\right)}\right\}^{1 / 2}
$$

$$
\begin{aligned}
& \cdot\left\{1+\frac{\lambda(1-\lambda)\left(1+\cos ^{2} \theta_{\nu, n}^{(\lambda)}\right)}{4\left[g_{n}^{(\lambda)}\left(\theta_{\nu, n}^{(\lambda)}\right)\right]^{2} \sin ^{4} \theta_{\nu, n}^{(\lambda)}}-\frac{3 \lambda^{2}(1-\lambda)^{2} \cos ^{2} \theta_{\nu, n}^{(\lambda)}}{4\left[g_{n}^{(\lambda)}\left(\theta_{\nu, n}^{(\lambda)}\right]^{3} \sin ^{6} \theta_{\nu, n}^{(\lambda)}\right.}\right\} \\
= & c_{n}^{(\lambda) G} \frac{\sin ^{2 i} \theta_{\nu, n}^{(\lambda)}\left\{\frac{(n+\lambda)^{2}+\lambda(1-\lambda)}{v_{4, n}(\pi / 2)}\right\}_{n}^{1 / 2}\left(\theta_{\nu, n}^{(\lambda)}\right)}{} \\
& \cdot\left\{1+\frac{\lambda(1-\lambda)\left(1+\cos ^{2} \theta_{\nu, n}^{(\lambda)}\right)}{4\left[g_{n}^{(\lambda)}\left(\theta_{\nu, n}^{(\lambda)}\right)\right]^{2} \sin ^{4} \theta_{\nu, n}^{(\lambda)}}+\nu^{-6} O(1)\right\} \\
= & c_{n}^{(\lambda) G} \sin ^{2 \lambda} \theta_{\nu, n}^{(\lambda)}\left\{1-\frac{\lambda(1-\lambda) \cos ^{2} \theta_{\nu, n}^{(\lambda)}}{(n+\lambda)^{2} \sin ^{2} \theta_{\nu, n}^{(\lambda)}+\lambda(1-\lambda)}\right\}^{1 / 2} \\
& \cdot\left\{1+\frac{\lambda(1-\lambda)\left(1+2 \cos ^{2} \theta_{\nu, n}^{(\lambda)}+(-1)^{n} \sin ^{4} \theta_{\nu, n}^{(\lambda)}\right)}{4(n+\lambda)^{4} \sin ^{4} \theta_{\nu, n}^{(\lambda)}}+\nu^{-6} O(1)\right\} .
\end{aligned}
$$

Applying (2.15) to this equation, we obtain (2.28). For the proof of (2.29) and (2.30) we only have to substitute the estimates (3.10) for $\theta_{\nu, n}^{(\lambda)}$ into (2.28). The last relation (2.31) in Theorem 4 follows from (2.28) using Lemma 5.

The corollaries can be shown by substituting the estimates of Lemma 1 for $c_{n}^{(\lambda)}$ into the respective estimates of Theorems 2 and 3 . The bounds $(2.26)$ in Remark 4 are obtained using, in addition, the bounds for the zeros of $P_{n}^{(i)}$ in (2.25). The calculations are lengthy but elementary. By (3.42 ff) the function $v_{3, n}^{(\lambda)}$ is monotonic in $(0, \pi / 2)$. Therefore, Remark 1 follows directly from the representation $(3.40)$.

\section{NUMERICAL EXAMPLES}

Using Lemmas 2 and 3, all estimates stated in the above theorems and corollaries remain bounded for all $\nu, n \in \mathbf{N}$ and fixed $\lambda \geq-\frac{4}{11}$. By results of Buell [7] it follows that this is also valid for $\lambda<-\frac{4}{11}$ if $\nu \neq 1$ and $\nu \neq n$. For the standard weight function $w \equiv 1$, i.e., $\lambda=\frac{1}{2}$, Table 1 indicates the precision of the bounds obtained, even for small $\nu$ and $n$. A further estimation using inequalities for the first nodes given by Szegö [35] and Gatteschi and Vinardi [15] shows that for this weight function the absolute value of the relative error of the lower bounds stated in Corollary 1 is smaller than $0.5 \%$ for all $n$ and $\nu$ and at most $0.05 \%$ and $0.005 \%$ for $1<\nu<n$ and $2<\nu<n-1$, respectively, while for the value $\bar{\varepsilon}_{\nu^{\prime}, n}^{(1 / 2)}$ in Corollary 2 we have by $(2.21)$ that $\bar{\varepsilon}_{1, n}^{(1 / 2)}<\frac{1}{30} \frac{1}{\nu^{4}}$ for $1 \leq \nu \leq(n+1) / 2$. 


\section{Applications}

For brevity, we restrict consideration to the standard weight function $w \equiv$ 1 , i.e., $\lambda=\frac{1}{2}$. Most of the proofs of the results below require additional investigations. Much more precise results, also for other values of $\lambda$, as well as the respective proofs will be given elsewhere.

1. Applying the above results, and modifying methods used in [13], it can be shown that the weights $a_{\nu, n}$ of every positive quadrature formula $Q_{n}$ of the type (1.2) (i.e., $a_{\nu, n} \geq 0$ for all $\nu$ ) having degree $\operatorname{deg}\left(Q_{n}\right) \geq 2 m-1$ satisfy the inequality

$$
a_{\nu, n}<\frac{\pi}{m}\left\{\left[1-x_{\nu, n}^{2}\right]^{1 / 2}+\frac{\pi}{m}\left|x_{\nu, n}\right|\right\} .
$$

This improves asymptotic results previously obtained for special positive interpolatory quadrature formulae (see Kütz [25]).

2. In order to investigate the numerical stability of quadrature formulae, one possible measure is the sum of the squares of the weights of $Q_{n}$, the so-called 'variance' of $Q_{n}$ (see, e.g., Chebyshev [8], Ostrowski [29], Allasia [3], and Katz [23]). Applying Corollary 2, it can be shown that

$$
\frac{\pi^{2}}{2} \frac{1}{n+1}<\sum_{\nu=1}^{n}\left(a_{\nu, n}^{G}\right)^{2}<\frac{\pi^{2}}{2} \frac{1}{n}, \quad n \geq 1 .
$$

This improves an asymptotic result of Kahaner [22]. Note that for every quadrature formula $Q_{n}$ the above sum has at least the value $4 / n$ if $\operatorname{deg}\left(Q_{n}\right) \geq 0$. The inequalities (5.2) show that the variance of the Gaussian formulae differs from the variance of such 'Chebyshev-type formulae' at most by a factor 1.24.

3. To estimate the error $R_{n}^{G}$ of the Gaussian formula $Q_{n}^{G}$ for integrands with low continuity, Stroud [32] proposed to investigate the so-called 'error coefficients' of low order of the Gaussian formulae (see also Davis and Rabinowitz [10, p. 292]). Numerical examples given in Stroud and Secrest [33] for small $n$ indicate that these are not much greater than those of the respective optimal formulae. This is confirmed by applying Theorem 2 to Peano kernels of small order. We obtain, e.g.,

$$
\left|R_{n}^{G}[f]\right| \leq \frac{\pi}{2 n+1} \operatorname{Var} f, \quad\left|R_{n}^{G}[f]\right| \leq \frac{\pi^{2}}{8 n} \max _{x \in[-1,1]}\left|f^{\prime}(x)\right|,
$$

where $\operatorname{Var} f$ is the variation of $f$. These error coefficients cannot be improved asymptotically as $n \rightarrow \infty$ (see [30]). Note that for the respective optimal formulae using $n$ nodes we have the error coefficient $1 / n$ instead of $\pi /(2 n+1)$ for the first class $\{f \mid \operatorname{Var} f \leq 1\}$ of functions, and also $1 / n$ instead of $\pi^{2} /(8 n)$ for the second class $\left\{f\left|f \in C^{1}[-1,1], \max _{x \in[-1,1]}\right| f^{\prime}(x) \mid \leq 1\right\}$ of functions considered in (5.3); see, e.g., Braß [5].

4. In Hämmerlin [21, Problem 2], Professor H. Braß conjectured that $R_{n}^{G}[f]$ $=O\left(n^{-2}\right)$ for every bounded convex function $f$ as $n \rightarrow \infty$. Applying Theorem 4 , we can prove the validity of this conjecture and thus show that the Gaussian 


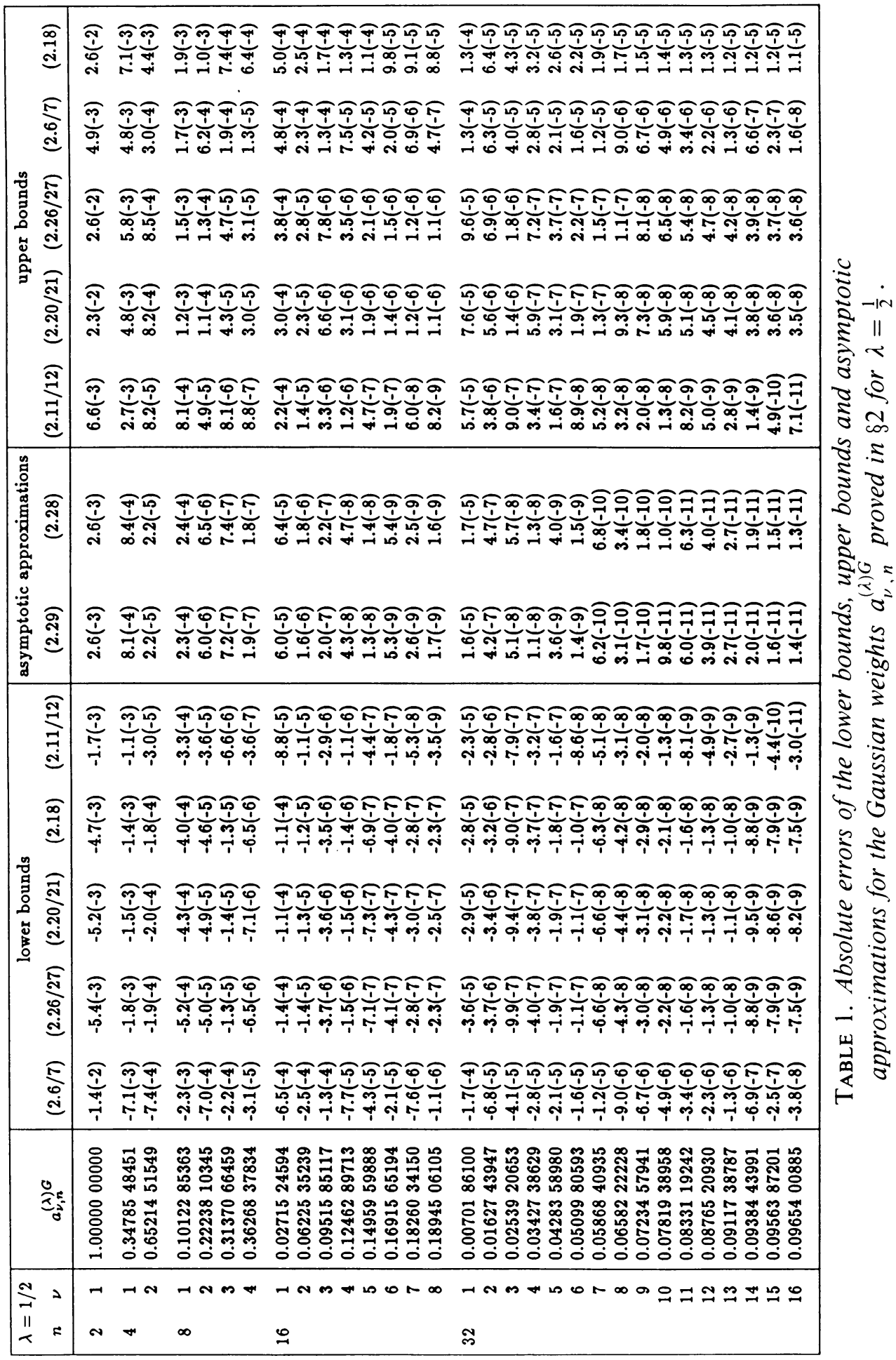


rule has asymptotically the best possible (see Braß [6]) order of convergence, even in this wide class of functions.

\section{ACKNOWLEDGMENT}

The authors are indebted to the referee for his helpful comments concerning stylistic and typographical errors in a previous version of this paper.

\section{BIBLIOGRAPHY}

1. M. Abramowitz and I. A. Stegun (eds.), Handbook of mathematical functions, 7th printing, Dover, New York, 1970.

2. S. Ahmed, M. E. Muldoon, and R. Spigler, Inequalities and numerical bounds for zeros of ultraspherical polynomials, SIAM J. Math. Anal. 17 (1986), 1000-1007.

3. G. Allasia, Proprietà statistiche delle formule di quadratura, Rend. Sem. Mat. Univ. Politec. Torino 35 (1976-77), 339-348.

4. S. N. Bernstein, Sur un système d'équations indéterminées, J. Math. Pures Appl. 17 (1938), 179-186.

5. H. Braß, Quadraturverfahren, Vandenhoeck \& Ruprecht, Göttingen, 1977.

6. ___ Zur Quadraturtheorie konvexer Funktionen, Numerical Integration (G. Hämmerlin, ed.), ISNM 57, Birkhäuser, Basel, 1982, pp. 34-47.

7. C. E. Buell, The zeros of Jacobi and related polynomials, Duke Math. J. 2 (1936), 304-316.

8. P. L. Chebyshev, Sur les quadratures, J. Math. Pures Appl. (2) 19 (1874), 19-34.

9. P. J. Davis and P. Rabinowitz, Some geometrical theorems for abscissas and weights of Gauss type, J. Math. Anal. Appl. 2 (1961), 428-437.

10. _ Methods of numerical integration, 2nd ed., Academic Press, London, 1984.

11. Á. Elbert, Some inequalities concerning Bessel functions of first kind, Studia Sci. Math. Hungar. 6 (1971), 277-285.

12. A. Erdélyi and F. G. Tricomi, The asymptotic expansion of a ratio of Gamma functions, Pacific J. Math. 1 (1951), 133-142.

13. K.-J. Förster, A comparison theorem for linear functionals and its applications in quadrature, Numerical Integration (G. Hämmerlin, ed.), ISNM 57, Birkhäuser, Basel, 1982, pp. 66-76.

14. K.-J. Förster and K. Petras, On the zeros of ultraspherical polynomials and Bessel functions (submitted).

15. L. Gatteschi and G. Vinardi, Sul grado di precisione di una formula di quadratura del tipo di Tchebycheff, Calcolo 15 (1978), 59-85.

16. L. Gatteschi, Una nuova rappresentazione asintotica dei polinomi ultrasferici, Calcolo $\mathbf{1 6}$ (1979), 447-458.

17. N New inequalities for the zeros of Jacobi polynomials, SIAM J. Math. Anal. 18 (1987), 1549-1562.

18. __ Uniform approximation of Christoffel numbers for Jacobi weight, Numerical Integration III (H. Braß and G. Hämmerlin, eds.), ISNM 85. Birkhäuser, Basel, 1988, pp. 49-59.

19. W. Gautschi, A survey of Gauss-Christoffel quadrature formulae, E. B. Christoffel, The Influence of his Work on Mathematics and the Physical Sciences (P. L. Butzer and F. Fehér, eds.), Birkhäuser Verlag, Basel, 1981, pp. 72-147.

20. A. Ghizzetti and A. Ossicini, Quadrature formulae, Academic Press, New York, 1970.

21. G. Hämmerlin (ed.). Numerical integration. ISNM 57. Birkhäuser Verlag. Basel, 1982.

22. D. K. Kahaner, Equal and almost equal weight quadrature formulas, SIAM J. Numer. Anal. 6 (1969), 551-556.

23. D. Katz, Optimal quadrature points for approximating integrals when function values are observed with errors, Math. Mag. 57 (1984). 284-290. 
24. V. I. Krylov, Approximate calculation of integrals (translated by A. H. Stroud), Macmillan, New York, 1962.

25. M. Kütz, On the asymptotic behaviour of some Cotes numbers, Z. Angew. Math. Mech. 66 (1986), 373-375.

26. H. N. Laden, Fundamental polynomials of Lagrange interpolation and coefficients of numerical quadrature, Duke. Math. J. 10 (1943), 141-151.

27. G. Monegato, Some new inequalities related to certain ultraspherical polynomials, SIAM J. Math. Anal. 11 (1980), 663-667.

28. A. Ossicini, Sulle costanti di Christoffel della formula di Gauss-Jacobi, Istit. Lombardo Accad. Sci. Lett. Rend. A 101 (1967), 169-180.

29. A. M. Ostrowski, On trends and problems in numerical approximation, On Numerical Approximation (R. Langer, ed.), Univ. of Wisconsin Press, Madison, Wisc., 1959, pp. 3-10.

30. K. Petras, Asymptotic behaviour of Peano kernels of fixed order, Numerical Integration III (H. Braß and G. Hämmerlin, ed.), ISNM 85, Birkhäuser, Basel, 1988, pp. 186-198.

31. J. A. Shohat and C. Winston, On mechanical quadratures, Rend. Circ. Mat. Palermo 58 (1934), 153-160.

32. A. H. Stroud, Estimating quadrature errors for functions with low continuity, SIAM J. Numer. Anal. 2 (1965), 480-488.

33. A. H. Stroud and D. Secrest, Gaussian quadrature formulas, Prentice-Hall, Englewood Cliffs, N.J., 1966.

34. G. Szegö, Asymptotische Entwicklungen der Jacobischen Polynome, Schriften der Königsberger Gelehrten Gesellschaft 10 (1933), 35-112. [Collected papers, vol. 2, pp. 401-477.]

35. __, Inequalities for the zeros of Legendre polynomials and related functions, Trans. Amer. Math. Soc. 39 (1936), 1-17. [Collected papers, vol. 2, pp. 593-609.]

36. __ Orthogonal polynomials, Amer. Math. Soc. Colloq. Publ., vol. 23, Amer. Math. Soc., Providence, R.I., 1939 (revised edition 1985).

37. F. G. Tricomi, Sugli zeri dei polinomi sferici ed ultrasferici, Ann. Mat. Pura Appl. 31 (1950), 93-97.

38. E. L. Whitney, Estimates of weights in Gauss-type quadrature, Math. Comp. 19 (1965), 277-286.

39. C. Winston, On mechanical quadrature formulae involving the classical orthogonal polynomials, Ann. of Math. (2) 35 (1934), 658-677.

Institut für Mathematik, Universität Hildesheim, D-3200 Hildesheim, Federal RepubLIC OF GermanY

Institut für Angewandte Mathematik, Technische Universität Braunschweig, D-3300 Braunschweig, Federal Republic of Germany 